\title{
Laboratório no estágio de gestão do SUS: integração ensino, pesquisa e gestão
}

\author{
Caroline Stein*; Cristine Maria Warmling**; Luísa Helena do Nascimento Tôrres***; Rafaela Soares

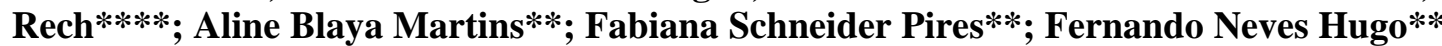

* Discente, Programa de Pós-Graduação em Odontologia, Saúde Bucal Coletiva, FO/UFRGS

** Docente, Departamento de Odontologia Preventiva e Social, FO/UFRGS

*** Docente, Departamento de Odontologia Preventiva e Social FO/UFSM

**** Discente, Programa de Pós-Graduação em Epidemiologia, FM/UFRGS

Recebido em 23/12/2017. Aprovado em 26/04/2018.

\section{RESUMO}

O estudo objetivou analisar a experiência de inovação do estágio de gestão no Sistema Único de Saúde (SUS), em um percurso formativo no curso de graduação de Odontologia, que integrou ensino, pesquisa e gestão. A operação-ensaio proporcionou uma contínua problematização de uma visão ampliada e transformadora da gestão. O laboratório constituiu-se em um campo do Estágio Curricular Supervisionado II da Odontologia da Faculdade de Odontologia da Universidade Federal do Rio Grande do Sul (FO-UFRGS). Os estudantes conheceram e discutiram aspectos de monitoramento e avaliação da Atenção Básica (AB) no Rio Grande do Sul (RS) com o Grupo de Trabalho do Departamento de Ações em Saúde da Secretaria Estadual de Saúde (DAS-RS). A partir de bancos de dados do Programa Nacional de Melhoria do Acesso e da Qualidade da Atenção Básica disponibilizados pelo Centro de Pesquisa em Odontologia Social da FO-UFRGS ao longo do semestre, os estudantes analisaram dados, produziram resultados e discutiram estratégias para a AB. Foi produzido um relatório final com as análises de variáveis relacionadas às Unidades e Equipes de $\mathrm{AB}$ de Saúde e as entrevistas com cirurgiões-dentistas das trinta Regiões de Saúde do RS. Ao final do semestre, foram apresentados os relatórios produzidos no Laboratório no Estágio de Gestão aos serviços e estabeleceu-se uma discussão sobre estratégias para a $\mathrm{AB}$ de modo a prover informações para a gestão e a tomada de decisão em saúde. Conclui-se que foi possível de modo simultâneo aprender, ensinar e prover informações para gestão e tomada de decisão no SUS.

Descritores: Ensino. Saúde Bucal. Gestão em Saúde. Ensino superior. Estágios. 


\section{INTRODUÇÃO}

A tarefa de planejar e realizar a gestão dos serviços de saúde que compõem o Sistema Único de Saúde (SUS) possibilita inovações políticas, gestoras e assistenciais ${ }^{1}$, assim como educacionais. As Instituições de Ensino Superior (IES) pelas diretrizes da Associação Brasileira de Ensino Odontológico (ABENO) devem realizar atividades de estágio curricular em serviços de saúde que oportunizem ações de planejamento e gestão ${ }^{2,3}$. Cada IES tem a liberdade de definir as dinâmicas de estágios curriculares visando a aproximação dos estudantes com a realidade social e epidemiológica do país, bem como com o planejamento do SUS.

Tradicionalmente, pela maior expansão da saúde bucal na Atenção Primária à Saúde, as IES direcionaram a inserção dos estudantes em estágios neste nível de atenção ${ }^{3,4}$. Inovações foram produzidas, muitas vezes, no próprio âmago das dificuldades encontradas para a consolidação dos estágios, como por exemplo, número de campos de estágios, ou a baixa cobertura da Estratégia Saúde da Família (ESF) no município. Diante disso, e visando a pluralidade de cenários e práticas, tem sido bemvinda a alternativa de ampliação de convênios com municípios próximos ao território da IES. Além disso, destaca-se que, com a Política Nacional de Saúde Bucal (PNSB) e a criação dos Centros de Especialidades Odontológicas (CEO), os cenários de estágios na atenção especializadas também emergiram como uma necessidade e oportunidade ${ }^{5}$.

A Faculdade de Odontologia da Universidade Federal do Rio Grande do Sul (FOUFRGS) priorizou nas reformas curriculares um tempo prolongado de vivências em ambientes de trabalho e itinerários das Redes de Atenção Primária e Especializada do SUS. O Estágio Curricular Supervisionado II da Odontologia (ESO II), cenário do estudo, ocorre no décimo semestre do curso e possui o objetivo de permitir ao estudante a compreensão de processos de trabalho em serviços de Atenção Especializada, Hospitalar e Gestão na Saúde Bucal do SUS ${ }^{6}$.

O Laboratório no Estágio de Gestão (LEG), experiência pedagógica que articula ensino, pesquisa e gestão, objeto de análise deste estudo, foi uma atividade desenvolvida no âmbito do ESO II com o objetivo de proporcionar aos estudantes, por meio de uma experiência de gestão do monitoramento e avaliação da Atenção Primária no estado do Rio Grande do Sul (RS), a oportunidade de desenvolvimento de competências de compreensão de políticas de gestão e planejamento no SUS.

Articulou-se o Centro de Pesquisa em Odontologia Social (CPOS) da FO-UFRGS, que desempenha um papel na colaboração e vinculação de projetos de pesquisa relacionados ao SUS $^{7}$ e estudantes do Programa de PósGraduação em Odontologia da área de concentração Saúde Bucal Coletiva. A parceria que o CPOS desenvolve com o Departamento de Ações em Saúde da Secretaria Estadual de Saúde (DAS) do RS permitiu o uso de dados oriundos do Primeiro Ciclo (2011-2012) do Programa Nacional de Melhoria do Acesso e da Qualidade da Atenção Básica (PMAQ-AB) ${ }^{8}$. Foi possível de modo simultâneo aprender, ensinar e prover informações para gestão e tomada de decisão.

Os Gestores das Regiões de Saúde do RS apresentaram demandas quanto à disponibilidade de informações de suas regiões. De saúde, tendo em vista que a rede de serviços, os relatórios do PMAQ-AB apresentam informações por estado, enquanto o portal do Ministério da Saúde (MS) fornece informações por equipe e por município. Além disso, o sistema do MS não dispõe de várias informações consideradas estratégicas pelos gestores e o LEG veio com o objetivo de supri-las. Neste sentido, a finalidade pedagógica do estágio visou responder a uma demanda do 
DAS-RS.

Neste contexto, o presente estudo possui o objetivo de analisar a experiência de inovação no estágio de gestão no SUS, em um percurso formativo no curso de graduação de Odontologia da FO-UFRGS, que integrou ensino, pesquisa e gestão.

\section{LABORATÓRIO NO ESTÁGIO DE GESTÃO DO SUS: OPERAÇÃO-ENSAIO}

O LEG efetivou-se a partir de uma nova experiência que foi se desenvolvendo ao longo do semestre, que denominou-se metodologicamente como operação-ensaio que, de acordo com Larrosa ${ }^{9}$, é o ensaio de uma determinada operação de "modo experimental do pensamento e da escrita que ainda pretende ser uma escrita pensante, pensativa, que ainda se produz como uma escrita que dá o que pensar", sem renunciar a uma constante reflexão sobre si mesma e a uma permanente metamorfose. Nesta perspectiva, esse método desenvolveu-se na forma experimental de ensaiar-se o pensamento e a escrita, que se encontrava em construção e em contínua problematização e reproblematização, durante todo $\mathrm{o}$ processo de planejamento $\mathrm{e}$ execução do LEG. A operação-ensaio, proporcionou um olhar ampliado e reflexivo aos docentes e discentes de possibilidades para a gestão, a partir da experiência da sua própria contingência e transformação. Com crítica, indagação, saber e atitude vai-se além da experiência para a construção de uma visão gestora mais ampliada e transformadora 9 .

2.1 Estágio Supervisionado De Odontologia II e Programa Nacional de Melhoria do Acesso e da Qualidade da Atenção Básica

O ESO II foca na atuação do formando na Atenção Especializada e busca propiciar aos estudantes do curso a inserção em serviços de Gestão e Atenção Especializada e hospitalar, dando continuidade ao Estágio Supervisionado de Odontologia I (ESO I), cujo foco está na vivência e atuação na Atenção Básica do município de Porto Alegre, RS.

De acordo com o Plano de Ensino ESO II, o estudante de Odontologia terá a oportunidade de vivenciar processos de trabalho nos diferentes serviços de Saúde Bucal do SUS, de Atenção Especializada, Hospitalar e Gestão. Tal estrutura permite a compreensão da composição, do funcionamento, da organização e da gestão dos processos de trabalho na Rede de Atenção em Saúde Bucal (RASB) no SUS em sua complexidade. O estudante é instrumentalizado a atuar nos cenários da RASB e desenvolve ações de apoio às instituições públicas que a compõem ${ }^{3,6}$. Além dos campos de atuação nos serviços, o estágio proporciona encontros presenciais semanais com tutorias, em que o estudante problematiza a realidade de trabalho em que está inserido, por meio de estudos de casos, elaboração, execução e avaliação de projetos, experiências de pesquisas e outros.

O campo de estágio LEG foi pensado e desenvolvido na ocasião de um estágio docente, que a partir da operação-ensaio, permitiu uma experiência modificadora e um trabalho crítico sobre o pensamento e planejamento da prática pedagógica. O Laboratório foi realizado sob a colaboração de professores e estudantes de pósgraduação. Os estudantes de pós-graduação participaram como tutores dos estudantes do ESO II, sob supervisão da equipe de docentes da disciplina.

A partir do CPOS/FO-UFRGS, que foi executor da avaliação externa do PMAQ-AB, obteve-se acesso aos dados utilizados no LEG. O PMAQ-AB foi instituído em 2011 com o objetivo de ampliar o acesso e o avanço da qualidade da atenção básica, com garantia de um padrão de qualidade comparável nacional, regional e localmente, de maneira a permitir 
maior transparência e efetividade das ações governamentais direcionadas à Atenção Básica em Saúde ${ }^{8}$.

Trata-se de uma avaliação de abrangência nacional executada de forma multicêntrica $\mathrm{e}$ integrada, por diversas instituições de ensino e pesquisa e com o acompanhamento direto do MS. Os resultados encontrados referem-se às avaliações respondidas pelos trabalhadores nas Unidades de Saúde, mediante entrevista e verificação de documentações in loco. Os dados utilizados no estágio aqui descrito são referentes à etapa de Avaliação Externa do Programa realizada com um instrumento padronizado e testado previamente, abrangendo três módulos com questões que avaliaram aspectos referentes à estrutura das Unidades Básicas de Saúde (UBS), aos processos de trabalho desenvolvidos pelas equipes de saúde e a satisfação dos usuários com os serviços de saúde ${ }^{10}$. A partir de dados do primeiro ciclo do PMAQ-AB, foi possível sua utilização para ocorrer o LEG e assim oportunizar uma nova possibilidade de campo de estágio de gestão.

\subsection{Laboratório no Estágio de Gestão}

Durante o semestre do curso, o LEG se desenvolveu a partir de etapas que possibilitaram aos estudantes se integrarem aos conceitos do PMAQ-AB, manusear dados em software estatístico e produzir um relatório com análise de diversas variáveis do banco de dados sobre as Unidades Básicas e Equipes de Saúde do estado do RS e processos de trabalho das Equipes de Saúde Bucal (ESB) para elaborar estratégias a partir dos resultados encontrados. Quinze estudantes foram divididos em 4 grupos de acordo com as 30 Regiões de Saúde (conforme proposto pelo DAS-RS), ficando cada grupo responsável por trabalhar com dados do PMAQAB de oito Regiões de Saúde. O estágio desenvolveu-se durante 14 semanas e suas atividades estão descritas no quadro 1 .
A partir das necessidades do DAS-RS e dos interesses dos estagiários, as variáveis analisadas por Região de Saúde do RS foram selecionadas: Módulo I: Tipo de Unidade de Saúde, Número de profissionais de saúde, Número de dentistas, se possuía sala de Acolhimento multiprofissional, se existia consultório odontológico, número de consultórios odontológicos, consultórios odontológicos com computador, consultórios odontológicos com computador conectado à internet; e Módulo II Ações de educação permanente que a equipe de saúde bucal participa, se a ESB realiza monitoramento e análise dos indicadores e informações de saúde bucal, o que a agenda da equipe de saúde bucal garantia, se a ESB garante agendamento de retorno para a continuidade do tratamento de um usuário que iniciou seu tratamento. Para cada grupo as demais questões foram de livre escolha e, portanto, variaram bastante entre as questões selecionadas.

A partir dos resultados encontrados para cada Região de Saúde, cada grupo de estudantes, realizou uma revisão de literatura, incluindo normas e manuais do que é preconizado pelo MS e elaborou estratégias e alternativas para os problemas encontrados, que pudessem orientar a tomada de decisão. Isto proporcionou aos estudantes exercerem a reflexão posicionando-se como gestores utilizando alternativas embasadas nas propostas pelo MS

Assim, a conclusão do estágio em gestão resultou em um Relatório final de que foi composto pelos seguintes tópicos: Introdução (contextualização do PMAQ-AB, definição de problemas e objetivos); Métodos (atividades desenvolvidas, variáveis analisadas e análise dos dados); Resultados (por Região de Saúde: características da Região, tabelas com resultados para o Módulo I e II PMAQ-AB, problemas identificados e estratégias propostas); Cronograma; Monitoramento das atividades; Conclusões e Referências. 


\begin{tabular}{|c|c|c|}
\hline Sem. & Atividade de Concentração na FO & Atividade não presencial \\
\hline 1 & $\begin{array}{l}\text { Apresentação inicial da proposta do LEG com explicação sobre } \\
\text { como se daria o desenvolvimento do LEG. Abordaram-se também } \\
\text { conceitos fundamentais sobre o PMAQ-AB e a contextualização da } \\
\text { utilização de seus dados pela gestão de modo a prover informações e } \\
\text { contribuir na tomada de decisão. As } 30 \text { Regiões de Saúde do RS } \\
\text { foram divididas entre } 4 \text { grupos de estudantes que ficaram sob } \\
\text { orientação de um tutor ao longo do estágio. }\end{array}$ & $\begin{array}{l}\text { Leitura do Manual PMAQ-AB }{ }^{8} \text { e Instrumento de } \\
\text { Avaliação Externa para as ESB }{ }^{10} \text {. }\end{array}$ \\
\hline 2 & Sem atividade presencial. & $\begin{array}{l}\text { Leitura de artigos relacionados ao PMAQ-AB, da Revista } \\
\text { Saúde em Debate }{ }^{11} \text {. }\end{array}$ \\
\hline 3 & $\begin{array}{l}\text { Roda de conversa sobre PMAQ-AB com representante do DAS-RS, } \\
\text { na oportunidade o DAS abordou demandas de interesse para gestão } \\
\text { e junto aos estudantes estabeleceu uma discussão sobre quais } \\
\text { variáveis do Instrumento de Avaliação das ESB analisariam. }\end{array}$ & $\begin{array}{l}\text { Acesso pela Plataforma de Ensino a Distância Moodle } \\
\text { UFRGS de Tutorial para orientações de elaboração do } \\
\text { Relatório do LEG e para download do software PSPP para } \\
\text { e trazer instalado em um computador portátil por grupo no } \\
\text { próximo encontro presencial. }\end{array}$ \\
\hline 4 & $\begin{array}{l}\text { Apresentação de Tutorial para conhecimento e desenvolvimento do } \\
\text { LEG através da análise de dados do PMAQ-AB, visando a } \\
\text { elaboração e interpretação de dados, que gerou um Relatório de } \\
\text { resultados para utilização na tomada de decisão nas Coordenadorias } \\
\text { Regionais de Saúde Bucal do estado do Rio Grande do Sul; } \\
\text { Capacitação e treinamento para utilização do software PSPP, para } \\
\text { análise dos dados. Disponibilização pelo CPOS de banco de dados } \\
\text { do Módulo } 1 \text { - Observação na Unidade Básica de Saúde (PMAQ- } \\
\text { AB). }\end{array}$ & 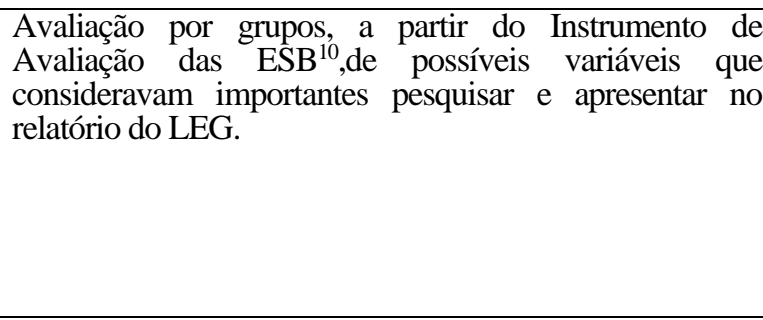 \\
\hline 5 & $\begin{array}{l}\text { Seleção das variáveis para análise, a partir do banco de dados e do } \\
\text { Instrumento de Avaliação Externa das ESB. }\end{array}$ & $\begin{array}{l}\text { Análise e elaboração de resultados preliminares, a partir } \\
\text { das primeiras variáveis selecionadas, relacionadas às } \\
\text { Unidades de Saúde e Equipes de AB, por Região de Saúde. }\end{array}$ \\
\hline 6 & $\begin{array}{l}\text { Elaboração de resultados do PMAQ-AB para cada Região de Saúde } \\
\text { com discussões e orientações para correç̃es e melhoramentos. }\end{array}$ & $\begin{array}{l}\text { Orientações e correções de apresentação de resultados } \\
\text { preliminares e envio por e-mail para correção. }\end{array}$ \\
\hline 7 & $\begin{array}{l}\text { Elaboração de resultados do PMAQ-AB para cada Região de Saúde } \\
\text { com discussões e orientações para correções e melhoramentos. }\end{array}$ & $\begin{array}{l}\text { Envio, aos estudantes, de novo banco de dados, Módulo } 2 \\
\text { - Entrevista com Profissional da Equipe de AB e } \\
\text { Verificação de Documentos na Unidade de Saúde }{ }^{10} \text { para } \\
\text { análise de novas variáveis. }\end{array}$ \\
\hline 8 & $\begin{array}{l}\text { Definição de novas variáveis incluídas, relacionadas a entrevista com } \\
\text { o CD. Em conjunto foram selecionadas novas variáveis e cada grupo } \\
\text { pode selecionar outras diferentes variáveis de interesse. Elaboração } \\
\text { de resultados do PMAQ-AB para cada Região de Saúde com } \\
\text { discussões e orientações para correções e melhoramentos. }\end{array}$ & $\begin{array}{l}\text { Análise de dados do Módulo } 2 \text { - Entrevista com } \\
\text { Profissional da Equipe de Atenção Básica e Verificação de } \\
\text { Documentos na Unidade de Saúde. }\end{array}$ \\
\hline 9 & $\begin{array}{l}\text { A partir dos resultados encontrados, cada grupo, juntamente com o } \\
\text { seu tutor, levantou e discutiu propostas de estratégias para a solução } \\
\text { dos problemas identificados a partir dos resultados. }\end{array}$ & $\begin{array}{l}\text { Elaboração de estratégias para a solução dos problemas } \\
\text { identificados a partir dos resultados, com orientação de } \\
\text { elaborar estratégias baseando-se no que é preconizado pelo } \\
\text { Ministério da Saúde. }\end{array}$ \\
\hline 10 & $\begin{array}{l}\text { Discussão e elaboração de propostas de estratégias para a solução dos } \\
\text { problemas identificados a partir dos resultados. }\end{array}$ & $\begin{array}{l}\text { Orientações e correções de apresentação de estratégias } \\
\text { propostas e envio por e-mail para os estudantes } \\
\text { aperfeiçoarem os relatórios. }\end{array}$ \\
\hline 11 & Sem atividade presencial. & $\begin{array}{l}\text { Orientações e correções de apresentação de estratégias } \\
\text { propostas e envio por e-mail para os estudantes } \\
\text { aperfeiçoarem os relatórios. }\end{array}$ \\
\hline 12 & $\begin{array}{l}\text { Com o grupo todo e, posteriormente, cada tutor com seu grupo } \\
\text { discutiu-se questões de finalização do Relatório do LEG, bem como } \\
\text { foram dadas orientações de correções e melhoramentos }\end{array}$ & $\begin{array}{l}\text { Finalização da escrita do Relatório do LEG e envio por e- } \\
\text { mail para os tutores. Elaboração de apresentação sobre o } \\
\text { mesmo. }\end{array}$ \\
\hline 13 & \multicolumn{2}{|c|}{$\begin{array}{l}\text { Apresentação do Relatório final de cada grupo para toda a turma do último semestre, destacando os resultados principais } \\
\text { encontrados, os problemas identificados e estabelecendo-se discussão sobre as estratégias propostas. }\end{array}$} \\
\hline 14 & \multicolumn{2}{|c|}{$\begin{array}{l}\text { Apresentação do Relatório final de cada grupo, com resultados e estratégias sobre o PMAQ-AB, para o DAS-RS. Neste encontro, } \\
\text { foi estabelecida uma discussão entre Equipe de trabalho do LEG, estudantes estagiários e representantes da DAS-RS. } \\
\text { Na oportunidade ocorreu uma reflexão sobre os resultados encontrados e estratégias propostas, tendo em vista o encontro dos } \\
\text { resultados apresentados pelos relatórios e a proximidade de contato com a realidade representada pela DAS-RS. Constituindo-se } \\
\text { em uma importante e necessária integração da pesquisa (dados do PMAQ-AB), do ensino (estudantes estagiários em fase de } \\
\text { formação) e da gestão (DAS-RS). }\end{array}$} \\
\hline
\end{tabular}

Sem. (semana); FO (Faculdade de Odontologia); LEG (Laboratório no Estágio de Gestão); PMAQ-AB (Programa Nacional de Melhoria do Acesso e da Qualidade da Atenção Básica); RS (Rio Grande do Sul); ESB (Equipe de Saúde Bucal); DAS (Departamento de Ações em Saúde da Secretaria Estadual de Saúde do Rio Grande do Sul); UFRGS (Universidade Federal do Rio Grande do Sul); ESB (Esquipes de Saúde Bucal); PSPP (Programa Estatístico livre/open source). 


\section{DISCUSSÃO}

O Estágio Curricular Supervisionado II, junto ao Centro de Pesquisa em Odontologia Social e o Departamento de Ações em Saúde da Secretaria Estadual de Saúde do Rio Grande do Sul, compôs uma estratégia viável de integração entre o ensino, pesquisa e gestão no SUS. Foi proporcionado aos estudantes a vivência na prática da construção, discussão e reflexão de ferramentas e estratégias de gestão da saúde. O LEG ampliou a formação profissional, permitindo, assim, o desenvolvimento de habilidade para a leitura e interpretação dos indicadores e dados coletados, tornando os estudantes mais aptos a tomar iniciativas, fazer o gerenciamento da força de trabalho, de recursos físicos e materiais e de informação, conforme preconizado nas DCN para a formação do $\mathrm{CD}^{12}$.

As inúmeras e constantes transformações da sociedade e a presença das novas $\mathrm{DCN}^{12}$, acrescidas das experiências na área têm reforçado a importância de discussão, reavaliação e inovação do processo de ensinoaprendizagem nos cursos de graduação de odontologia $^{13}$. A presente operação-ensaio descreve um estágio que se propõe não apenas realizar a replicação de habilidades e saberes que respondam a problemas específicos na área da gestão, mas também oportuniza a reflexão e experiência do estudante no próprio papel como futuro profissional frente aos problemas a serem enfrentados.

Compreendendo que em outro estudo, os estudantes demonstraram insatisfação com os campos de estágio de gestão ${ }^{5}$, nesta experiência ocorreu o resgate de conhecimentos já obtidos nos semestres anteriores do curso e que foram utilizados na elaboração do relatório final. Além disso, permitiu o entendimento e a identificação de diferentes realidades das experiências vividas nos estágios nos serviços de $\mathrm{AB}$ e especializada na capital, em relação às situações avaliadas das diversas Regiões de Saúde do estado, o que possibilitou a capacidade crítica a partir do encontro entre a teoria e a prática epidemiológica.

A aprendizagem de gestão na formação em saúde bucal proporcionou aos estagiários uma visão ampliada das condições estruturais das Unidades Básicas de Saúde e dos processos de trabalhos dos CD das 30 Regiões de Saúde do Estado do RS. Isto significou considerar para a avaliação e gestão um olhar sobre as necessidades encontradas e a possibilidade de reflexão e formulação de estratégias de planejamento, conforme proposto pelo quadrilátero da formação na área da saúde que ainda apresenta desafios para seu estabelecimento, principalmente em relação a valorização e inclusão do controle social na educação para saúde ${ }^{14}$.

Ainda, aos pós-graduandos envolvidos, o LEG contribuiu para solidificar conhecimentos de metodologias pedagógicas perante o contato e a discussão com os estudantes. Apresentou-se a experiência com uma dupla função: de aprender sobre os dados referentes às 30 Regiões de Saúde do Estado do RS, especialmente quanto aos aspectos ligados às ESB, e do ensino de análises, interpretações, e confecção de relatórios com linguagem facilitada, assim como, a difusão de informação de forma simples.

Do ponto de vista de sua função educativa, a universidade representa o local permanente do exercício da crítica. As experiências oportunizam uma herança cultural de saberes, pensamentos e valores que se propagam em cada estudante ${ }^{15}$. Criticar significa discernir, separar e julgar. É um ato que preserva o que merece ser afirmado e põe em dúvida a pretensão daquilo que pode ir além do seu domínio de aplicação e, portanto, não merece ser afirmado $^{16}$. Assim, experiências como desta operação-ensaio permitem refletir e criar tanto individualmente quanto coletivamente 
estratégias de enfrentamento aos desafios a serem encontrados. Além disso, tem-se a formação de um profissional com senso crítico capaz de planejar e ser agente de mudanças.

\section{CONCLUSÕES}

O LEG configurou-se em uma iniciativa pioneira no país e de caráter inovador na área pedagógica para a gestão, e pode-se concluir que obteve sucesso na sua conclusão. Foi uma experiência inovadora no campo da formação em saúde pois aproximou os estudantes de graduação com a gestão e o planejamento, construiu interfaces entre a graduação e a pósgraduação, além dos campos como a pesquisa e a gestão do SUS na esfera da graduação. Para a aquisição de habilidades e competências na formação em saúde como indicado nas DCN do curso de Odontologia, outros e novos olhares para o papel do estudante, dos tutores $\mathrm{e}$ preceptores na área da saúde devem ser discutidos. Qualificar a formação na direção de egressos com espirito crítico, com capacidade para tomada de decisões e liderança não se dará pela exaustão em conteúdos teóricos, mas sim pela riqueza da troca de experiências, pela construção compartilhada de projetos entre a instituição de ensino e os serviços de saúde e principalmente pela ousadia de inovar e redesenhar continuamente as possibilidades para a formação. Espera-se que este percurso possa contribuir na formação dos profissionais da saúde, ser divulgado e utilizado em outras IES da Odontologia e da área da saúde como uma nova proposta de aprendizado, integrando ensino, pesquisa e gestão no Sistema Único de Saúde.

\section{AGRADECIMENTOS}

Ao Centro de Pesquisa em Odontologia Social que oportunizou o acesso aos dados do Programa Nacional de Melhoria do Acesso e da Qualidade da Atenção Básica (Ministério da Saúde) e ao
Departamento de Ações em Saúde da Secretaria Estadual de Saúde do Rio Grande do Sul pela parceria da integração ensino-gestão.

\section{ABSTRACT \\ Laboratory in the management internship in SUS: integration of teaching, research and management}

The study aimed to analyze the innovation experience of the management internship in the Unified Health System (SUS), in a training course in the graduation of Dentistry, which integrated teaching, research and management. The operation essay provided a continuous problematization of an expanded and transformative view of management. The laboratory was constituted in a field of the Supervised Curricular Internship II of Dentistry of the Faculty of Dentistry of the Federal University of Rio Grande do Sul (FOUFRGS). The students met and discussed aspects of monitoring and evaluation of Primary Care (PC) in Rio Grande do Sul (RS) with the Working Group of the Department of Health Actions of the State Department of Health (DAS-RS). Based on databases of the National Program for Improving Access and Quality of Primary Care provided by the Center for Research in Social Dentistry of FO-UFRGS during the semester, students analyzed data, produced results and discussed strategies for PC. A final report was produced with analyzes of variables related to Health Units and Teams of PC and the interviews with dentists of the 30 Health Regions of RS. At the end of the semester, the reports produced in the Laboratory in the Service Management Internship were presented and a discussion on strategies for the PC was established in order to provide information for health management and decision making. It was concluded that it was possible simultaneously to learn, teach and provide information for management and decision making in SUS.

Descriptors: Teaching. Oral Health. Health Management. Education, Higher. Internships. 


\section{REFERÊNCIAS}

1. Carnut L, Narvai PC. Avaliação de desempenho de sistemas de saúde e gerencialismo na gestão pública brasileira. Saude e Soc. 2016;25(2):290-305.

2. Scavuzzi AIF, de Gouveia CVD, Carcereri DL, Veeck EB, Ranali J, da Costa LJ, Morita MC, de Araújo ME. Revisão das Diretrizes da ABENO para a definição do Estágio Supervisionado Curricular nos cursos de Odontologia. Rev ABENO. 2015;15(3) :109-13.

3. Warmling CM, Rossoni E, Hugo FN, Toassi RFC, Lemos VA, Maria S, et al. Estágios curriculares no SUS: experiências da Faculdade de Odontologia da UFRGS. Rev ABENO. 2011;11(2):63-70.

4. Morita MC, Kriger L. Mudanças nos cursos de Odontologia e a interação com o SUS. Rev ABENO. 2004;4(1):17-21.

5. Warmling CM, Baldisserotto J, Stocker J, Gallo DB, Hugo FN. O agir em competência para o cuidado especializado na saúde bucal. Rev ABENO. 2015;15(1):1-16.

6. Universidade Federal do Rio Grande do Sul. Plano de Ensino do Estágio Curricular Supervisionado II da Odontologia. Faculdade de Odontologia; 2016.

7. Universidade Federal do Rio Grande do Sul. Centro de Pesquisas em Odontologia Social. [Acesso em 19 dez. 2017]. Disponivel em: http://www.ufrgs.br/cpos/o-cpos/apresentacao

8. Brasil. Programa Nacional de Melhoria do Acesso e da Qualidade da Atenção Básica (PMAQ) - Manual Instrutivo. 2012. p. 66.

9. Larrosa J. A Operação Ensaio. Educ Realid. 2004;29(1):27-43.

10. Brasil. PMAQ - Instrumento de Avaliação Externa para as Equipes de Saúde Bucal. Brasília: Ministério da Saúde; 2013. p. 50.

11. Brasil. Saúde mais perto de você - Acesso e Qualidade - Programa Nacional de Melhoria do Acesso da Qualidade da Atenção Básica (PMAQ/AB). Brasília: Ministério da Saúde; 2013. p. 38.

12. BRASIL. Diretrizes Curriculares Nacionais do Curso de Graduação em Odontologia [Internet]. Vol. 1, Minstério da Saúde. 2002. p. 1-5. [Acesso em 19 dez. 2017]. Disponivel em: http://portal.mec.gov.br/cne/arquivos /pdf/CES032002.pdf

13. Secco LG, Pereira MLT. Concepções de qualidade de ensino dos coordenadores de graduação: uma análise dos cursos de odontologia do Estado de São Paulo. Interface (Botucatu). 2004;8(15):313-30.

14. Ceccim RB, Feuerwerker LCM. O quadrilátero da formação para a Área da Saúde: ensino, gestão, atenção e controle social. PHYSIS. 2004;14(1):41-65.

15. Morin E. Complexidade e transdisciplinaridade: a reforma da universidade e do ensino fundamental. Natal: EDUFRN; 1999.

16. Dictionary E. Online Etymology Dictionary. 2017 [Acesso em 19 dez. 2017]. Disponivel em: http://www.etymonline.com/index.php? $\underline{\text { allowed_in frame }=0 \& \text { search }=\text { critic }}$

\section{Correspondência para:}

Caroline Stein

e-mail: csteinodonto@hotmail.com

Universidade Federal do Rio Grande do Sul, Faculdade de Odontologia

Rua Ramiro Barcelos, 2492

90035-003 Porto Alegre/RS 